Check for updates

Cite this: RSC Adv., 2017, 7, 22875

Received 10th February 2017

Accepted 11th April 2017

DOI: 10.1039/c7ra01701d

rsc.li/rsc-advances

\title{
Atomically thin SiC nanoparticles obtained via ultrasonic treatment to realize enhanced catalytic activity for the oxygen reduction reaction in both alkaline and acidic media $\uparrow$
}

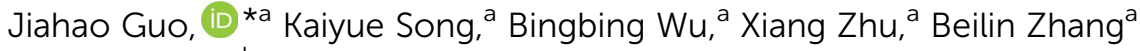 \\ and Yantao Shi ${ }^{\mathrm{b}}$
}

\begin{abstract}
Inorganic graphene analogues (IGAs) are promising catalysts for the oxygen reduction reaction (ORR) in fuel cells; however, their catalytic activities need to be improved. Herein, we report atomically thin $\mathrm{SiC}$ obtained via a simple ultrasonic treatment of commercial $\mathrm{SiC}$ as an efficient electrocatalyst for the ORR in alkaline and acidic media. Due to the advantages of its unique surface composition and structure, the atomically thin $\mathrm{SiC}$ shows remarkable catalytic activity for the ORR and the number of electrons transferred per $\mathrm{O}_{2}$ is close to four in both alkaline and acidic media. Compared with commercial $\mathrm{Pt} / \mathrm{C}$, the atomically thin $\mathrm{SiC}$ possesses prominent durability and tolerance to methanol crossover, and thus can be developed into a low-cost and efficient alternative as a cathodic electrode in fuel cells.
\end{abstract}

\section{Introduction}

The aggravation of environmental pollution requires new energy resources coupled with storage techniques to substitute for fossil fuels such as coal, petroleum, and natural gas. ${ }^{1,2}$ Fuel cells and batteries are considered to be very promising energy storage and conversion devices. ${ }^{3}$ One of the most important problems encountered by these new energy storage and conversion devices is the sluggish kinetic processes of the oxygen reduction reaction (ORR). ${ }^{4}$ Great efforts have been made in catalysis research to overcome the intrinsic ORR disadvantages. ${ }^{5-8}$ In recent years, despite the tremendous progress in the development of Pt-based catalytic materials, it is still a major challenge to develop highly active catalysts. Even if Pt-based catalysts are considered to be the best catalysts for the ORR in fuel cells, they still suffer from multiple disadvantages, such as high cost, limited reserves, and the crossover effect of fuel molecules..$^{9-11}$ In order to promote the commercialization of fuel cells, the development of nonprecious metal or metal-free ORR catalysts has attracted great interest. ${ }^{12-16}$

\footnotetext{
${ }^{a}$ College of Chemistry and Materials Engineering, Anhui Science and Technology University, Fengyang, Anhui, 233100, P. R. China. E-mail: guojiahao1974@163.com ${ }^{b}$ State Key Laboratory of Fine Chemicals, School of Chemistry, Dalian University of Technology, Dalian, 116024, P. R. China

$\uparrow$ Electronic supplementary information (ESI) available: SEM, TEM, HRTEM, AFM images of commercial SiC; O 1s, C 1s, Si 2p core-level of commercial SiC; binding energy and percentages of different bindings calculated from the deconvoluted XPS spectra of C 1s, Si 2p, and O 1s peak; CVs of commercial SiC. See DOI: 10.1039/c7ra01701d
}

Graphene, which has a two-dimensional layer structure, has attracted considerable attention because of its unique physical and chemical properties that exhibit significant advances in energy storage and conversion devices. ${ }^{17}$ In the area of electrocatalysis, graphene has been recognized as a promising material and has been modified via various methods for use in ORR catalysts. ${ }^{16-23}$ Inorganic graphene analogues (IGAs) such as $\mathrm{MoS}_{2}, \mathrm{MoSe}_{2}$, and $\mathrm{WSe}_{2}$ also belong to two-dimensional layered materials, which have similar properties with graphene. These materials generally possess large surface areas and large amounts of coordination-unsaturated surface atoms, which provide outstanding catalytic properties and are conducive for the adsorption of reactants. ${ }^{24}$ Xie $e t$ al. showed that defect-rich $\mathrm{MoS}_{2}$ ultrathin nanosheets exhibit excellent hydrogen evolution reaction catalytic activity with a small onset potential, large current density, small Tafel slope, and prominent electrochemical durability. ${ }^{25}$ Our group confirmed the excellent ORR activity of atomically thin $\mathrm{MoSe}_{2}$ /reduced graphene oxide and $\mathrm{WSe}_{2} /$ reduced graphene oxide nanosheets associated with the number of exchanged electrons close to four. ${ }^{26}$ As a typical IGA, silicon carbide ( $\mathrm{SiC}$ ) possesses low density, high thermal conductivity, good chemical stability, and high saturated carrier mobility, which result in wide applications in various fields. ${ }^{27-30}$ SiC with an atomic ratio of $1: 1$ has an imbalance of charge distribution on different atoms caused by the different electronegativities of Si (1.90) and C (2.55), which leads to more active sites than those by doped carbon materials that serve as ORR catalysts. Based on density functional theory (DFT), Zhang et al. exploited the potential of layered $\mathrm{SiC}$ as an ORR catalyst in alkaline media. ${ }^{31}$ However, no research on the electrocatalytic 
activity of layered $\mathrm{SiC}$ for the ORR and its preparation has been reported to date.

In this study, we highlight a simple pathway to prepare atomically thin $\mathrm{SiC}$ nanosheets derived from commercial $\mathrm{SiC}$ through ultrasonic exfoliation. Systematic characterization of its morphology, structure, composition and catalytic activity for the ORR in alkaline and acidic media is conducted. Compared with commercial SiC, the atomically thin SiC presents excellent catalytic activity for the ORR in both alkaline and acidic media, which is comparative to that of $\mathrm{Pt} / \mathrm{C}$ catalysts.

\section{Experimental}

\subsection{Preparation of atomically thin SiC nanomaterial}

Commercial SiC (300 mg) was added in a mixture of deionized water $(125 \mathrm{~mL})$ and anhydrous ethanol $(125 \mathrm{~mL})$ in a $500 \mathrm{~mL}$ flask. The mixed system was sealed and placed in a low-power ultrasonic bath (KQ3200DA) for $12 \mathrm{~h}$. The resultant dispersion was centrifuged at $2000 \mathrm{rpm}$ for $20 \mathrm{~min}$, and the supernatant was collected. The as-obtained product was collected after the supernatant, centrifuged at $12000 \mathrm{rpm}$ for $20 \mathrm{~min}$, washed with deionized water and anhydrous ethanol successively multiple times, and finally dried at $50{ }^{\circ} \mathrm{C}$ under vacuum overnight for further characterization.

\subsection{Materials characterization}

$\mathrm{X}$-ray diffraction (XRD) patterns were collected using a Rigaku $\mathrm{D} /$ max-rA X-ray diffractometer with $\mathrm{Cu} \mathrm{K} \alpha$ radiation $(\lambda=1.5418$ A). Field emission scanning electron microscopy (FESEM) images were obtained using an FEI HITACHI S-4800 FESEM. Transmission electron microscopy (TEM) images, highresolution TEM (HRTEM) images, and selected-area electron diffraction (SAED) patterns were obtained using a JEOL-2010 TEM with an acceleration voltage of $200 \mathrm{kV}$. Atomic force microscopy (AFM) was performed using a Veeco DI NanoScope MultiMode V system. The Brunauer-Emmett-Teller (BET) surface area was determined via nitrogen adsorption on a Tristar II 3020 Micromeritics adsorption analyzer at $77 \mathrm{~K}$. Prior to the adsorption measurements, the samples were degassed at $200{ }^{\circ} \mathrm{C}$ for a minimum of $10 \mathrm{~h}$. X-ray photoelectron spectra (XPS) were acquired on an ESCALAB MKII with $\mathrm{Mg} \mathrm{K} \alpha(h \nu=1253.6$ $\mathrm{eV}$ ) as the excitation source. The binding energies obtained in the XPS spectral analysis were corrected for specimen charging by referencing $\mathrm{C} 1 \mathrm{~s}$ to $284.5 \mathrm{eV}$.

\subsection{Electrochemical measurements}

The ORR activity of the atomically thin $\mathrm{SiC}$ was evaluated using a standard three-electrode electrochemical cell on an electrochemical workstation (CHI 660C, Chenhua, Shanghai) at room temperature. To prepare the working electrode, a homogeneous catalyst ink was made by mixture of $4 \mathrm{mg}$ catalyst, deionized water, isopropanol, and Nafion ionomer solution ( $5 \mathrm{wt} \%)(\mathrm{v} / \mathrm{v} / \mathrm{v}$ $=2.5: 1: 0.06$ ) to form a $4 \mathrm{mg} \mathrm{mL}^{-1}$ suspension, which was ultrasonicated for approximately $1 \mathrm{~h}$.

Furthermore, $5 \mu \mathrm{L}$ of catalyst slurry was spread onto the surface of a glassy carbon (GC) disk electrode $\left(0.2 \mathrm{~cm}^{2}\right)$ to form a catalyst loading of $\sim 100 \mu \mathrm{g} \mathrm{cm}^{-2}$ and was dried under ambient conditions. The electrochemical properties of the catalyst were measured in $0.1 \mathrm{M} \mathrm{KOH}$ or $0.1 \mathrm{M} \mathrm{HClO}_{4}$ aqueous solution. The modified GC electrode, a Pt plate, and an $\mathrm{Ag} / \mathrm{AgCl}$ electrode were used as the working, counter, and reference electrodes, respectively. The $\mathrm{Ag} / \mathrm{AgCl}$ reference electrode was calibrated against the reversible hydrogen electrode (RHE). Before testing, an $\mathrm{O}_{2} / \mathrm{N}_{2}$ flow was passed through the electrolyte in the cell for $30 \mathrm{~min}$ to saturate it with $\mathrm{O}_{2} / \mathrm{N}_{2}$. The ORR catalytic activity was determined using the rotating disk electrode (RDE) and rotating ring disk electrode (RRDE) techniques with linear sweep voltammetry (LSV) from 0 to $1.1 \mathrm{~V}$ (vs. RHE) at a scan rate of $10 \mathrm{mV} \mathrm{s}^{-1}$ in an $\mathrm{O}_{2}$-saturated $0.1 \mathrm{M} \mathrm{KOH}$ or $0.1 \mathrm{M} \mathrm{HClO}_{4}$ solution. Cyclic voltammetry curves $(\mathrm{CV})$ were collected in the potential range of 0 to $1.2 \mathrm{~V}(v s$. RHE) at a sweep rate of $10 \mathrm{mV}$ $\mathrm{s}^{-1}$. In this study, all the potentials were normalized to the RHE, all current densities were normalized to the BET surface area of the catalyst and all electrochemical experiments were carried out at room temperature and ambient pressure. Steady-state polarization and methanol crossover measurements were performed on the atomically thin $\mathrm{SiC}$ and $\mathrm{Pt} / \mathrm{C}$ using the same setup as $\mathrm{CV}$ in $\mathrm{O}_{2}$-saturated $0.1 \mathrm{M} \mathrm{KOH}$ or $0.1 \mathrm{M} \mathrm{HClO}_{4}$.

\section{Results and discussions}

\subsection{Material characterization}

The XRD patterns of the atomically thin SiC and commercial SiC are shown in Fig. 1a. There are four main diffraction peaks at $34.90^{\circ}, 40.52^{\circ}, 59.35^{\circ}$, and $71.12^{\circ}$, which can be indexed to the lattice planes (111), (200), (220), and (311) of cubic SiC (JCPDS card no. 29-1129), respectively. There is a small peak at $33.12^{\circ}$ (marked with an asterisk), which is likely due to the stacking faults within the crystal. ${ }^{32}$ The diffraction peaks are strong and sharp, which indicate that the atomically thin SiC possesses good crystallinity.

The FESEM images of the atomically thin SiC and commercial SiC are shown in Fig. $1 \mathrm{~b}$ and $\mathrm{S} 1 \mathrm{a}, \uparrow$ respectively. As shown in Fig. S1a, $\uparrow$ the commercial SiC is composed of both dispersed nanosized particles and some aggregates. The sizes of the

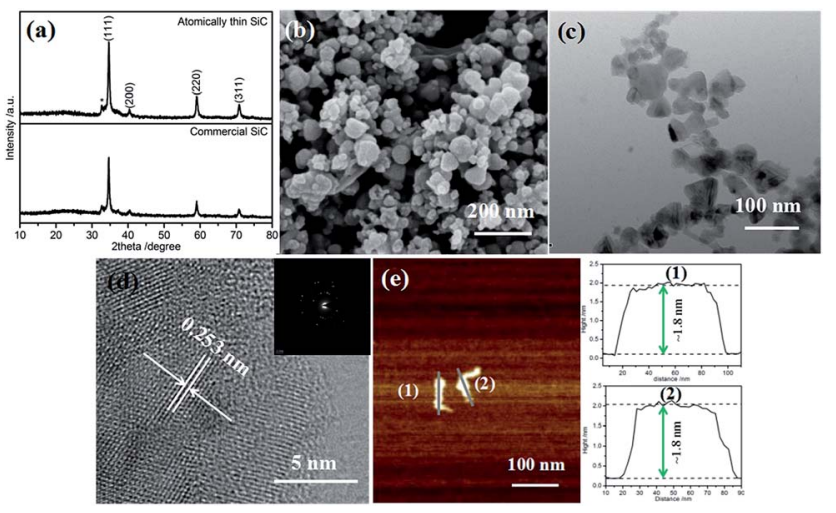

Fig. 1 (a) XRD, (b) SEM, (c) TEM, (d) HRTEM, and (e) AFM images and height profiles from the sections indicated by gray lines of the atomically thin SiC. The inset in (d) shows the corresponding SAED pattern. 
nanoparticles range from $50 \mathrm{~nm}$ to $170 \mathrm{~nm}$. After ultrasonic treatment, the nanoparticles of the atomically thin SiC are significantly reduced and no aggregates are observed, and its particle size is between 20 and $80 \mathrm{~nm}$. The decrease in particle size can cause an increase in the number of catalytic activity sites in SiC and thus improve its catalytic activity. Fig. 1c and $\mathrm{S} 1 \mathrm{~b} \dagger$ depict the TEM images of the atomically thin SiC and commercial SiC. Compared to the commercial SiC, the irregular nanoparticles of the atomically thin SiC are much smaller and their sizes range from $23 \mathrm{~nm}$ to $75 \mathrm{~nm}$. As can be seen from Fig. 1d, the lattice fringe spacing of the atomically thin $\mathrm{SiC}$ is measured to be $\sim 0.253 \mathrm{~nm}$, which is in good agreement with the value obtained using the Bragg diffraction from the (111) planes of $\beta$-SiC (Fig. 1c). ${ }^{33}$ The SAED patterns in Fig. 1d display diffraction points rather than diffraction circles, which further reveals that the atomically thin SiC exhibits an exfoliated thin layer. To confirm the thickness of the as-prepared sample, AFM imaging was carried out and the corresponding height profiles are shown in Fig. 1e. As can be seen in Fig. 1e, the atomically thin $\mathrm{SiC}$ possesses a relatively irregular, smooth $2 \mathrm{D}$ sheet structure with an average height of approximately $1.8 \mathrm{~nm}$. These results confirm that the commercial $\mathrm{SiC}$ was effectively exfoliated via ultrasonic treatment and an ultrathin nanomaterial was successfully obtained. This atomically thin structure can effectively improve the electrical conductivity, conducive to the charge transfer. Furthermore, it also facilitates reactant adsorption and increases the reactive sites, resulting in excellent catalytic activity.

The specific surface area and porous nature of the atomically thin SiC were measured via nitrogen adsorption/desorption measurements conducted at $77 \mathrm{~K}$. Compared with commercial $\mathrm{SiC}$, the isotherms of the atomically thin SiC can be classified as type-III with a distinct hysteresis loop at relative pressures $\left(P / P_{0}\right)$ from 0.45 to 1.0 (Fig. 2a), suggesting the presence of mesopores that can facilitate oxygen adsorption and molecular transportation during the ORR. The atomically thin SiC has a huge Brunauer-Emmett-Teller surface area of $53.85 \mathrm{~m}^{2} \mathrm{~g}^{-1}$ with an average pore diameter of $34 \mathrm{~nm}$ and pore volume of $0.365 \mathrm{~cm}^{3}$ $\mathrm{g}^{-1}$, which are higher than the commercial SiC. These results clearly illustrate that with a simple ultrasonic treatment, commercial SiC can be effectively exfoliated into atomically thin $\mathrm{SiC}$ with a thickness of only $1.8 \mathrm{~nm}$, which possesses a higher surface area.

The surface composition of the electrocatalysts has an important influence on their catalytic performances. The
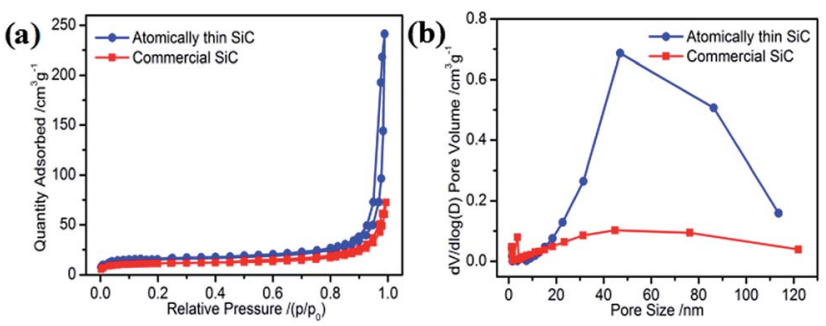

Fig. 2 (a) Nitrogen adsorption-desorption isotherms and (b) pore size distribution curves of the atomically thin $\mathrm{SiC}$ and commercial SiC. surface element composition of SiC was investigated via XPS. Fig. 3a shows the full-range XPS spectra of the atomically thin $\mathrm{SiC}$ and commercial SiC. As shown, the photoelectron peaks at $103.08 \mathrm{eV}, 154.08 \mathrm{eV}$, and $284.08 \mathrm{eV}$ can be indexed to Si $2 \mathrm{p}, \mathrm{Si}$ $2 \mathrm{~s}$, and $\mathrm{C} 1 \mathrm{~s}$, respectively. Oxygen can be detected for $\mathrm{SiC}$ on the basis of its binding energy. The presence of $\mathrm{O}$ can be attributed to the adsorption of oxygen molecules on the surface of SiC and further result in a strong effect between $\mathrm{Si}$ or $\mathrm{C}$ and $\mathrm{O}$. The unsaturated chemical bonds on the surface of the atomically thin SiC obtained by ultrasonic treatment should be responsible for this phenomenon, and resulting in the combination of $\mathrm{C}$ or $\mathrm{Si}$ and $\mathrm{N}$ on the surface of SiC. ${ }^{34}$ Fig. 3 and S2 $\uparrow$ show the corelevel XPS spectra of C 1s, Si 2p, N 1s, and O 1s and the corresponding deconvoluted spectra for the atomically thin SiC and commercial SiC. Based on the deconvoluted XPS spectra, the percentages and binding energies of the different groups are shown in Table S1. $\dagger$ From Fig. 3b, the deconvoluted peaks with binding energies of 283.7, 284.6, 285.3 and $286.2 \mathrm{eV}$ can be assigned to the $\mathrm{C}-\mathrm{Si}, \mathrm{C}-\mathrm{C}, \mathrm{C}-\mathrm{O}$ and $\mathrm{C}-\mathrm{N}$ bonds, respectively. ${ }^{35,36}$ The $\mathrm{C}-\mathrm{C}$ and $\mathrm{C}-\mathrm{Si}$ bonds are associated with carbon and $\mathrm{SiC}$ on the surface, respectively. The $\mathrm{C}-\mathrm{O}$ and $\mathrm{C}-\mathrm{N}$ bonds are attributed to the adsorption of $\mathrm{O}_{2}$ or $\mathrm{N}_{2}$. The $\mathrm{N}$ content is very low due to the low activity of $\mathrm{N}_{2}$. Compared with commercial SiC, the binding energies of the $\mathrm{C}-\mathrm{Si}, \mathrm{C}-\mathrm{C}$, and $\mathrm{C}-\mathrm{O}$ bonds increase in different degrees. The increase in binding energy is beneficial for the adsorption and dissociation of $\mathrm{O}_{2}$ on the surface of the catalyst, which promotes the catalytic activity of the atomically thin SiC. The deconvoluted peak with the binding energy of $288.9 \mathrm{eV}$ for commercial SiC can be assigned to the $\mathrm{COO}$ bond, which indicates that the commercial SiC surface contains more oxygen and is not conducive to oxygen adsorption. The deconvolution of the corresponding $\mathrm{Si} 2 \mathrm{p}$ spectrum for the atomically
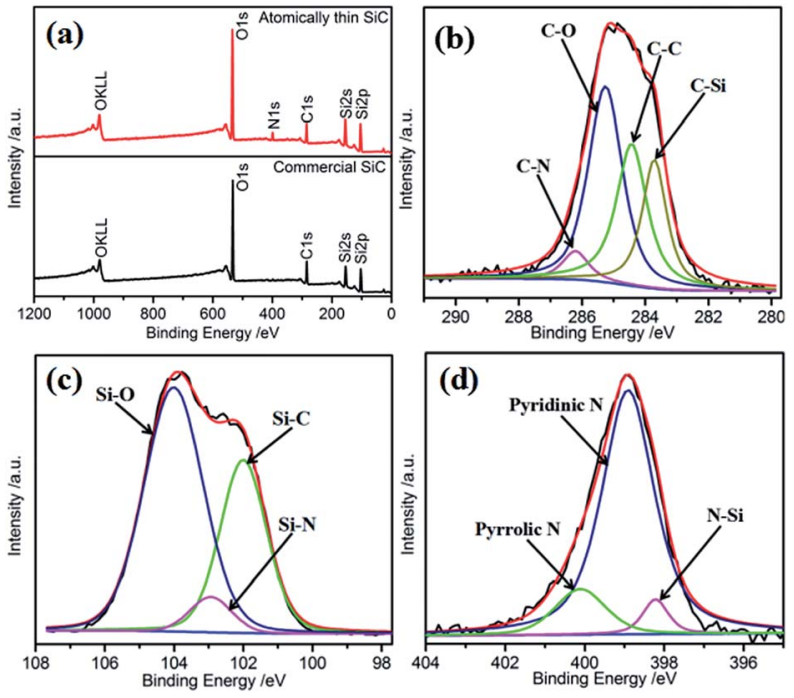

Fig. 3 (a) Overall XPS spectra of the atomically thin SiC and commercial SiC; (b) C 1s core-level and corresponding deconvoluted spectra for the atomically thin $\mathrm{SiC}$; (c) $\mathrm{Si} 2 \mathrm{p}$ core-level and corresponding deconvoluted spectra for the atomically thin $\mathrm{SiC}$; and (d) N 1s core-level and corresponding deconvoluted spectra for the atomically thin SiC. 
thin SiC (Fig. 3c) indicates that three chemical bonding states, $\mathrm{Si}-\mathrm{C}, \mathrm{Si}-\mathrm{N}$, and $\mathrm{Si}-\mathrm{O}$, are on its surface. ${ }^{37}$ The appearance of the $\mathrm{Si}-\mathrm{N}$ bond is consistent with previous results. The binding energies of $\mathrm{Si}-\mathrm{C}$ and $\mathrm{Si}-\mathrm{O}$ are located at 102.1 and $104.0 \mathrm{eV}$, respectively, which are also higher than the corresponding bonds in commercial SiC. The content of $\mathrm{Si}-\mathrm{O}$ bonds is significantly higher than that of $\mathrm{Si}-\mathrm{C}$ bonds, which indicates that $\mathrm{Si}$ mainly exists in the form of $\mathrm{Si}-\mathrm{O}$ on the surface of $\mathrm{SiC}$, and $\mathrm{O}_{2}$ can be easily adsorbed and dissociated on the Si sites. As can be seen from Fig. S2d, $\uparrow \mathrm{O}-\mathrm{C}$ and $\mathrm{O}-\mathrm{Si}$ bonds are detected for the atomically thin SiC, and their binding energies are higher than that of commercial SiC. Compared with that of commercial SiC, the fraction of $\mathrm{O}-\mathrm{Si}$ bonds increases from $47.4 \%$ to $63.0 \%$, and the fraction of $\mathrm{O}-\mathrm{C}$ bonds significantly decreases from $52.6 \%$ to $37.0 \%$ for the atomically thin SiC. The O-Si bond with the binding energy of $533.2 \mathrm{eV}$ corresponds to amorphous $\mathrm{SiO}_{2} \cdot{ }^{38}$ The XPS results show that the binding energies of the atomically thin SiC are higher than that of commercial SiC because of its surface unsaturated bonds, which are convenient for the adsorption and dissociation of $\mathrm{O}_{2}$ on the surface of the catalyst. The adsorbed oxygen mainly exists in the form of amorphous $\mathrm{SiO}_{2}$. Fig. 3d displays the high-resolution $\mathrm{N}$ 1s XPS spectrum of the atomically thin SiC sample as well as the corresponding deconvolution spectra of the different types of nitrogen probably existing in the sample. The asymmetric $\mathrm{N}$ 1s spectrum indicates the existence of three types of $\mathrm{N}$ : $\mathrm{N}-\mathrm{Si}$ at $398.2 \mathrm{eV}$, pyridinic $\mathrm{N}$ at $398.9 \mathrm{eV}$, and pyrrolic $\mathrm{N}$ at $400.1 \mathrm{eV} .{ }^{39}$ The content of pyridinic $\mathrm{N}$ is as high as $80.9 \%$. The pyridinic $\mathrm{N}$ and pyrrolic $\mathrm{N}$ atoms are located at the edge or defect sites, ${ }^{\mathbf{4 0}}$ which indicates that the ultrasonic treatment is responsible for the existence of $\mathrm{N}$. Pyridinic $\mathrm{N}$ atoms, as a marker of plane exposure, can provide actual active sites and improve the ORR activity of the atomically thin SiC. ${ }^{\mathbf{4 1 , 4 2}}$

\subsection{Electrocatalytic analyses}

First, the ORR catalytic activity of the atomically thin SiC in alkaline medium was investigated. Cyclic voltammetry measurements were conducted in $\mathrm{O}_{2^{-}}$or $\mathrm{N}_{2}$-saturated $0.1 \mathrm{M}$ $\mathrm{KOH}$ solution to evaluate the ORR activities of the atomically thin SiC (Fig. 4a). There is no visible peak in the plot of the measurements in $\mathrm{N}_{2}$ saturated $\mathrm{KOH}$ solution, which indicates that the oxygen reduction reaction did not occur. Nevertheless, a well-defined oxygen reduction peak at $0.76 \mathrm{~V}$ ( $v s$. RHE) appeared in the presence of $\mathrm{O}_{2}$, which indicates that $\mathrm{SiC}$ possesses ORR catalytic activity in alkaline electrolyte. Compared with commercial SiC, the atomically thin SiC possesses a higher reduction peak potential and larger cathodic current, which suggest higher catalytic activity for the ORR.

To further investigate its ORR performance, linear sweep voltammetry (LSV) measurements on an RDE for the atomically thin SiC, commercial SiC, and Pt/C electrodes were carried out in $\mathrm{O}_{2}$-saturated $0.1 \mathrm{M} \mathrm{KOH}$ solution at a scan rate of $10 \mathrm{mV} \mathrm{s}^{-1}$ and a rotation rate of $1600 \mathrm{rpm}$. As shown in Fig. 4b, the ORR based on the commercial SiC catalyst commences around $0.83 \mathrm{~V}$, followed by a continuous increase in current density with no plateau. The onset potential for oxygen reduction at the
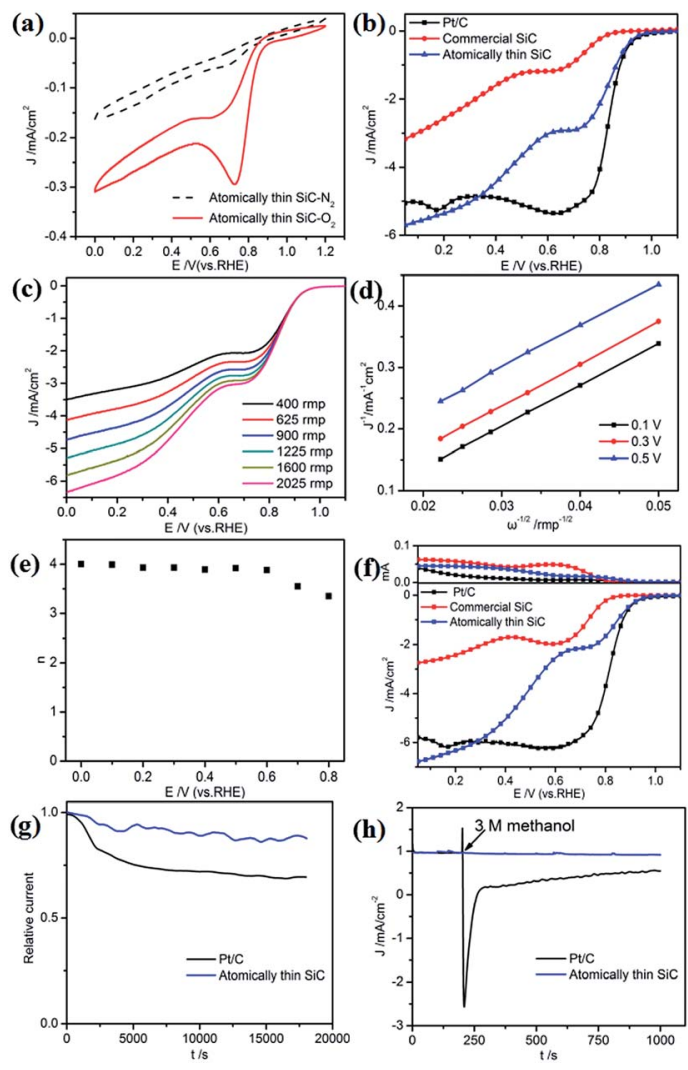

Fig. 4 (a) CVs of the atomically thin $\mathrm{SiC}$ in $\mathrm{N}_{2}$ - and $\mathrm{O}_{2}$-saturated $0.1 \mathrm{M}$ $\mathrm{KOH}$ solution at a scan rate of $10 \mathrm{mV} \mathrm{s}^{-1}$. (b) LSV of the atomically thin $\mathrm{SiC}$, commercial $\mathrm{SiC}$, and $\mathrm{Pt} / \mathrm{C}$ in $\mathrm{O}_{2}$-saturated $0.1 \mathrm{M} \mathrm{KOH}$ at a scan rate of $10 \mathrm{mV} \mathrm{s}^{-1}$ with an RDE rotation rate of $1600 \mathrm{rpm}$. (c) LSV of the atomically thin $\mathrm{SiC}$ at different $\mathrm{RDE}$ rotation rates. (d) Calculated $\mathrm{K}-\mathrm{L}$ plots of the ORR from the atomically thin SiC. (e) Electron-transfer number $n$ derived from the $K-L$ plots at different potentials. (f) RRDE from the atomically thin $\mathrm{SiC}$. (g) Chronoamperometric response $(i-t)$ of the atomically thin $\mathrm{SiC}$ and $\mathrm{Pt} / \mathrm{C}$ and (h) $i-t$ of the atomically thin $\mathrm{SiC}$ and $\mathrm{Pt} / \mathrm{C}$ before and after the addition of $3 \mathrm{M}$ methanol. Tests were conducted in $\mathrm{O}_{2}$-saturated $0.1 \mathrm{M} \mathrm{KOH}$ solution at $0.7 \mathrm{~V}$.

atomically thin SiC electrode significantly shifts positively to $0.95 \mathrm{~V}$, which is close to the onset potential for $\mathrm{Pt} / \mathrm{C}$ electrodes $(0.97 \mathrm{~V})$. The limiting diffusion current on the atomically thin SiC electrode at $0.3 \mathrm{~V}$ becomes about 2.5 times stronger with a relatively wide plateau with respect to the commercial $\mathrm{SiC}$ electrode, which indicates a diffusion-controlled process related to an efficient $4 \mathrm{e}^{-}$dominated ORR pathway. ${ }^{43-45}$ For the atomically thin SiC electrode, its LSV curve exhibits two current plateaus at $0.8-0.6 \mathrm{~V}$ and below $0.6 \mathrm{~V}$, respectively, corresponding to a two-electron process and four-electron process, respectively. This result may affect the catalytic activity of the atomically thin SiC. The results of CV and LSV prove that the atomically thin SiC electrode shows favorable ORR catalytic activity, which is reflected by its positive onset potential and high current density.

RDE voltammetry measurements were also carried out to gain further insight into the ORR performance of the atomically thin SiC. Fig. 4c shows the RDE current-potential curves at different rotation rates for the atomically thin $\mathrm{SiC}$ in an alkaline 
medium. In order to obtain the number of electrons transferred per oxygen molecule $(n)$ on the atomically thin SiC, K-L plots from the LSVs at various rotation speeds were calculated from the Koutecky-Levich equations as follows: ${ }^{46-48}$

$$
\begin{gathered}
\frac{1}{J}=\frac{1}{J_{\mathrm{K}}}+\frac{1}{J_{\mathrm{L}}}=\frac{1}{J_{\mathrm{K}}}+\frac{1}{B \omega^{1 / 2}} \\
B=0.2 n C_{\mathrm{O}}\left(D_{\mathrm{O}}\right)^{2 / 3} \nu^{-1 / 6}
\end{gathered}
$$

where $J, J_{\mathrm{K}}$ and $J_{\mathrm{L}}$ are the measured, kinetic, and diffusionlimiting current density, respectively. $\omega$ is the rotation speed in rpm. $n$ is the number of electrons transferred during ORR process, $F$ is the Faraday constant $\left(F=96485 \mathrm{C} \mathrm{mol}^{-1}\right), D_{\mathrm{O}}$ is the diffusion coefficient of $\mathrm{O}_{2}, C_{\mathrm{O}}$ is the bulk concentration of $\mathrm{O}_{2}$, and $\nu$ is the kinetic viscosity of the electrolyte. In this study, the electrolyte is $\mathrm{O}_{2}$-saturated $0.1 \mathrm{M} \mathrm{KOH}$. The $D_{\mathrm{O}}, C_{\mathrm{O}}$ and $\nu$ values used are $1.9 \times 10^{-5} \mathrm{~cm}^{2} \mathrm{~s}^{-1}, 1.2 \times 10^{-6} \mathrm{~mol} \mathrm{~cm}^{-3}$, and $0.01 \mathrm{~cm}^{2} \mathrm{~s}^{-1}$, respectively. The constant 0.2 in eqn (2) is adopted when the rotation speed is expressed in rpm. Fig. 4d shows three linear K-L plots at different potentials, which suggest that the inverse current density $(1 / j)$ is a function of the inverse of the square root of the rotation speed $\left(\omega^{-1 / 2}\right)$ at different potential values and first order reaction kinetics toward the concentration of $\mathrm{O}_{2}$ on the atomically thin $\mathrm{SiC}$ from $0.1 \mathrm{~V}$ to $0.5 \mathrm{~V}$. As shown in Fig. 4e, the corresponding numbers of electrons transferred were calculated to be $3.7-4.0$ per $\mathrm{O}_{2}$ molecule at 0$0.6 \mathrm{~V}$ for the atomically thin SiC. The $n$ value is lower at high potential. This result shows that the ORR process at the atomically thin SiC electrode is dominated by a four-electron process with water as the product at low potential. Fig. $4 \mathrm{f}$ shows the rotation ring-disk electrode (RRDE) curves of the three catalysts in $\mathrm{O}_{2}$-saturated $0.1 \mathrm{M} \mathrm{KOH}$ solution at a scan rate of $10 \mathrm{mV} \mathrm{s}^{-1}$ and rotation rate of $1600 \mathrm{rpm}$. The atomically thin SiC exhibits a much higher diffusion-limiting current density than that of commercial SiC. The RRDE measurements show that the $\mathrm{HO}_{2}{ }^{-}$ yield of the atomically thin SiC is less than $9 \%$, which is significantly lower than commercial SiC (Fig. $4 \mathrm{f}$ and S3†). The number of electrons transferred is calculated to be 3.6-3.9 over the potential range of $0.1-0.8 \mathrm{~V}$. This is well consistent with the result obtained from the $\mathrm{K}-\mathrm{L}$ plots based on $\mathrm{RDE}$ measurements.

Regarding the potential use of a catalyst in direct methanol fuel cells, its stability and tolerance to methanol crossover are key parameters to evaluate its performance. The durability of the atomically thin SiC and commercial Pt/C for the ORR was evaluated by continuous chronoamperometric measurements at $0.7 \mathrm{~V}$ in $\mathrm{O}_{2}$-saturated $0.1 \mathrm{M} \mathrm{KOH}$ solution (Fig. $4 \mathrm{~g}$ ). The ORR current density of the atomically thin SiC decreases more slowly than that of commercial Pt/C, maintaining $91 \%$ of its initial current density even after $18000 \mathrm{~s}$. This result indicates that the atomically thin SiC demonstrates better stability than commercial Pt/C in alkaline medium. The high stability of the atomically thin $\mathrm{SiC}$ is mainly derived from its thin layer structure, which ensures no significant decrease in catalytic active sites on its surface. Moreover, the catalytic selectivity of the atomically thin $\mathrm{SiC}$ was determined from its chronoamperometric responses at the potential of $0.7 \mathrm{~V}$ in $0.1 \mathrm{M}$ $\mathrm{KOH}$ solution with $3 \mathrm{M}$ methanol added (Fig. $4 \mathrm{~h}$ ). For comparison, the corresponding chronoamperometric response for the $\mathrm{Pt} / \mathrm{C}$ catalyst was also tested. No significant response is observed in the ORR current for the atomically thin SiC catalyst. However, a sharp decrease in the ORR current can be observed for the Pt/C catalyst after the addition of methanol. This result indicates that the atomically thin SiC catalyst has higher selectivity toward the ORR and better methanol tolerance than the commercial Pt/C in an alkaline medium, which is relevant in practical applications.

The atomically thin SiC was also used as a catalyst for the ORR in acidic medium. The electrocatalytic activity of the atomically thin $\mathrm{SiC}$ in $0.1 \mathrm{M} \mathrm{HClO}_{4}$ was investigated via $\mathrm{CV}$ (Fig. 5a). Compared with $\mathrm{N}_{2}$ saturated solution, the $\mathrm{CV}$ curve in $\mathrm{O}_{2}$ saturated $0.1 \mathrm{M} \mathrm{HClO}_{4}$ solution presents a remarkable ORR current peak at $0.62 \mathrm{~V}$ (vs. RHE) and a larger cathodic current comparable with commercial SiC appears, which indicate high ORR catalytic activity in acidic medium.
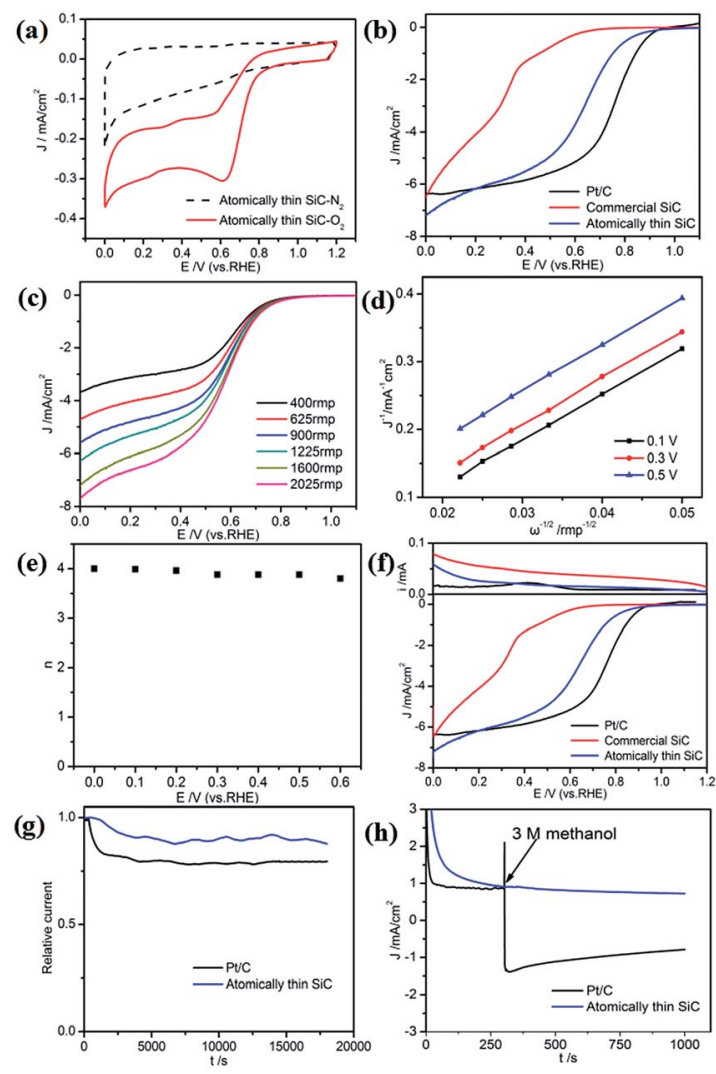

Fig. 5 (a) $\mathrm{CV}$ s of the atomically thin $\mathrm{SiC}$ in $\mathrm{N}_{2}$ - and $\mathrm{O}_{2}$-saturated $0.1 \mathrm{M}$ $\mathrm{HClO}_{4}$ solution at a scan rate of $10 \mathrm{mV} \mathrm{s}^{-1}$. (b) LSV of the atomically thin $\mathrm{SiC}$, commercial $\mathrm{SiC}$, and $\mathrm{Pt} / \mathrm{C}$ in $\mathrm{O}_{2}$-saturated $0.1 \mathrm{M} \mathrm{HClO}_{4}$ at a scan rate of $10 \mathrm{mV} \mathrm{s}^{-1}$ with an RDE rotation rate of $1600 \mathrm{rpm}$. (c) LSV of the atomically thin $\mathrm{SiC}$ at different RDE rotation rates. (d) Calculated $\mathrm{K}-\mathrm{L}$ plots of the ORR on the atomically thin SiC. (e) Electron-transfer number $n$ derived from the $K-L$ plots at different potentials. (f) RRDE curves of the atomically thin $\mathrm{SiC}$. (g) Chronoamperometric response $(i-t)$ of the atomically thin $\mathrm{SiC}$ and $\mathrm{Pt} / \mathrm{C}$ and $(\mathrm{h}) i-t$ of the atomically thin $\mathrm{SiC}$ and $\mathrm{Pt} / \mathrm{C}$ before and after the addition of $3 \mathrm{M}$ methanol. Tests were conducted in $\mathrm{O}_{2}$-saturated $0.1 \mathrm{M} \mathrm{HClO}_{4}$ solution at $0.7 \mathrm{~V}$. 
The ORR performance was characterized by LSV measurements on an $\mathrm{RDE}$ in $\mathrm{O}_{2}$-saturated $0.1 \mathrm{M} \mathrm{HClO}_{4}$ solution. As seen from Fig. 5b, commercial SiC shows weaker catalytic activity and lower onset potential in an acidic medium. The ORR onset potential for the atomically thin SiC commenced at around $0.88 \mathrm{~V}$, which is close to $\mathrm{Pt} / \mathrm{C}$ electrodes $(0.92 \mathrm{~V})$. The limiting diffusion current on the atomically thin SiC electrode presents a relatively wide plateau with respect to the commercial SiC electrode.

To estimate the number of electrons transferred for the atomically thin SiC in acidic medium, LSV and K-L plots at different potentials were obtained according to the $\mathrm{K}-\mathrm{L}$ equations, which are shown in Fig. $5 \mathrm{c}$ and $\mathrm{d}$. The values of $D_{\mathrm{O}}, C_{\mathrm{O}}$ and $\nu$ are $1.93 \times 10^{-5} \mathrm{~cm}^{2} \mathrm{~s}^{-1}, 1.26 \times 10^{-6} \mathrm{~mol} \mathrm{~cm}{ }^{-3}$, and 0.01 $\mathrm{cm}^{2} \mathrm{~s}^{-1}$, respectively in $0.1 \mathrm{M} \mathrm{HClO}_{4}$ solution. ${ }^{49}$ The three curves show a good linear relationship and the corresponding number of electrons transferred is 3.8-4.0 per $\mathrm{O}_{2}$ molecule at different potentials for the atomically thin SiC. According to the RRDE results obtained from $0.1 \mathrm{M} \mathrm{HClO}_{4}$ solution (Fig. 5e), the atomically thin SiC also exhibits a much higher diffusionlimiting current density than that of commercial SiC. The RRDE measurements show that the $\mathrm{HO}_{2}{ }^{-}$yield of the atomically thin SiC is less than 7\%, which is significantly lower than that of commercial SiC (Fig. 5f and S3 $\dagger$ ). The number of electrons transferred is calculated to be 3.7-4.0 over the potential range of 0-0.6 V. The results of the electrochemical experiments prove that the atomically thin SiC electrode shows favorable ORR catalytic activity in acidic media. Moreover, we speculate that the catalytic activity of the atomically thin $\mathrm{SiC}$ is also reduced by the solubility of acid and can be further improved by changing the structure of the catalyst.

The stability and tolerance to methanol crossover of the atomically thin $\mathrm{SiC}$ in acidic medium were also evaluated using the same method. Compared with the performance of commercial Pt/C, the attenuation of the ORR current density for the atomically thin $\mathrm{SiC}$ is evidently slow (Fig. $5 \mathrm{~g}$ ). Its selectivity toward the ORR and methanol tolerance are better than that of commercial Pt/C in an acidic medium (Fig. 5h).

The ORR performance of the atomically thin $\mathrm{SiC}$ is mainly attributed to its ultrathin structure and the presence of a small amount of nitrogen. These microstructure and composition characteristics facilitate reactant adsorption, increase the reactive sites and improve the electrical conductivity of the atomically thin SiC, which lead to an improvement in the catalytic activity.

\section{Conclusions}

In summary, atomically thin $\mathrm{SiC}$ is obtained using an ultrasonic exfoliation method and used as an ORR catalyst in fuel cells. The particle size of SiC considerably decreases, its surface area increases, and the surface of the atomically thin SiC contains numerous unsaturated bonds and a small amount of nitrogen, which are favorable for the adsorption and dissociation of reactants. The ultrathin structure of the atomically thin SiC significantly improves the ORR catalytic activity in both alkaline and acidic media. The number of electrons transferred for the
ORR on the atomically thin $\mathrm{SiC}$ catalyst is close to 4 , which indicates that a four-electron reduction pathway is favored. The atomically thin SiC also exhibits outstanding durability and methanol crossover resistance in both alkaline and acidic media. This study provides a new idea for the design and application of non-precious ORR catalysts.

\section{Acknowledgements}

Financial support was provided by the Natural Science Foundation of Anhui Province (KJ2016A171), the Stable Talent Foundation of Anhui Science and Technology University (HCWD201601), the Anhui college students innovation and entrepreneurship training program (201610879048), and Materials Science and Engineering Key Discipline foundation (AKZDXK2015 A01).

\section{Notes and references}

1 Y. G. Guo, J. S. Hu and L. J. Wan, Adv. Mater., 2008, 20, 2878.

2 M. L. Xiao, J. B. Zhu, L. G. Feng, C. P. Liu and W. Xing, $A d v$. Mater., 2015, 27, 2521-2527.

3 M. Winter and R. J. Brodd, Chem. Rev., 2004, 104, 4245.

4 E. M. Ericson, M. S. Thorum, R. Vasic, N. S. Marinkovic, A. L. Frenkel, A. A. Gewirth and R. G. Nuzzo, J. Am. Chem. Soc., 2012, 134, 197-200.

5 C. H. Choi, M. W. Chung, H. C. Kwon, S. H. Park and S. I. Woo, J. Mater. Chem. A, 2013, 1, 3694-3699.

6 Z. Y. Mo, H. L. Peng, H. G. Liang and S. J. Liao, Electrochim. Acta, 2013, 99, 30-37.

7 S. Y. Wang, D. S. Yu, L. M. Dai, D. W. Chang and J. B. Baek, ACS Nano, 2011, 5, 6202-6209.

8 S. Y. Wang, D. S. Yu and L. M. Dai, J. Am. Chem. Soc., 2011, 133, 5182-5185.

9 N. Hoshi, M. Nakamura and A. Hitotsuyanagi, Electrochim. Acta, 2013, 112, 899-904.

10 J. Chen, B. Lim, E. P. Lee and Y. Xia, Nano Today, 2009, 4(1), 81-95.

11 T. Yu, D. Y. Kim, H. Zhang and Y. Xia, Angew. Chem., Int. Ed., 2011, 50, 2773-2777.

12 G. Wu, K. L. More, C. M. Johnston and P. Zelenay, Science, 2011, 332, 443-447.

13 E. Proietti, F. Jaouen, M. Lefvre, N. Larouche, J. Tian, J. Herranz and J. P. Dodelet, Nat. Commun., 2011, 2, 416-424.

14 H. W. Liang, W. Wei, Z. S. Wu, X. L. Feng and K. Mullen, J. Am. Chem. Soc., 2013, 135, 16002-16005.

15 L. J. Yang, S. J. Jiang, Y. Zhao, L. Zhu, S. Chen, X. Z. Wang, Q. Wu, J. Ma, Y. W. Ma and Z. Hu, Angew. Chem., Int. Ed., 2011, 50, 7132-7135.

16 Z. H. Sheng, H. L. Gao, W. J. Bao, F. B. Wang and X. H. Xia, J. Mater. Chem., 2012, 22, 390-395.

17 D. Chen, L. Tang and J. Li, Chem. Soc. Rev., 2010, 39, 31573180.

18 L. Qu, Y. Liu, J. B. Back and L. M. Dai, ACS Nano, 2010, 4, 1321-1326.

19 Z. Y. Lin, G. Waller, Y. Liu, M. L. Liu and C. P. Wong, Adv. Energy Mater., 2012, 2, 884-888. 
20 Z. J. Jiang, Z. Q. Jiang and W. H. Chen, J. Power Sources, 2014, 251, 55-65.

21 H. J. Kim, K. Lee, S. I. Woo and Y. Jung, Phys. Chem. Chem. Phys., 2011, 13, 17505-17510.

22 Z. H. Sheng, L. Shao, J. J. Chen, W. J. Bao, F. B. Wang and X. H. Xia, ACS Nano, 2011, 5, 4350-4358.

23 J. E. Park, Y. J. Jang, Y. J. Kim, M. S. Song, S. H. Yoon, D. H. Kim and S. J. Kim, Phys. Chem. Chem. Phys., 2014, 16, 103-109.

24 Y. F. Sun, F. C. Lei, S. Gao, B. C. Pan, J. F. Zhou and Y. Xie, Angew. Chem., Int. Ed., 2013, 52, 10569.

25 J. F. Xie, H. Zhang, S. Li, R. X. Wang, X. Sun, M. Zhou, J. F. Zhou, X. W. Lou and Y. Xie, Adv. Mater., 2013, 25, 5807-5813.

26 J. H. Guo, Y. T. Shi, X. G. Bai, X. C. Wang and T. L. Ma, J. Mater. Chem. A, 2015, 3, 24397-24404.

27 R. B. Wu, K. Zhou, C. Y. Yue, J. Wei and Y. Pan, Prog. Mater. Sci., 2015, 72, 1-30.

28 Y. W. Kim, K. Y. Lim and W. S. Seo, J. Am. Ceram. Soc., 2014, 97, 923-928.

29 M. Herrmann, K. Sempf, M. Schneider, U. Sydow, K. Kremmer and A. Michaelis, J. Eur. Ceram. Soc., 2014, 34, 229-235.

30 A. M. Kueck, Q. M. Ramasse, L. C. De Jonghe and R. O. Riche, Acta Mater., 2010, 58, 2999-3005.

31 P. Zhang, B. B. Xiao, X. L. Hou, Y. F. Zhu and Q. Jiang, Sci. Rep., 2014, 4, 3821-3828.

32 R. B. Wu, Z. H. Yang, M. S. Fu and K. Zhou, J. Alloys Compd., 2016, 687, 833-838.

33 W. Liu, L. F. Cheng, Y. G. Wang and H. J. Ma, J. Eur. Ceram. Soc., 2016, 36, 3901-3907.

34 J. H. Boo, M. Kim, S. B. Lee, S. J. Park and J. G. Han, J. Vac. Sci. Technol., A, 2000, 18, 1713-1717.
35 E. Beche, M. Balat-Pichelin, V. Flaud, J. Esvan, T. Duguer, D. Sciti and D. Alfano, Surf. Interface Anal., 2014, 46, 817822.

36 Y. Mizokawa, K. Geib and C. Wilmsen, J. Vac. Sci. Technol., A, 1986, 4, 1696-1700.

37 A. Stoch, J. Stoch and A. Rakowska, Surf. Interface Anal., 1994, 22, 242-247.

38 Y. S. Jin, Q. J. Yan, Z. R. Yin and Y. Chen, J. Chem. Soc., Faraday Trans., 1995, 91, 381-384.

39 H. T. Liu, Y. Q. Liu and D. B. Zhu, J. Mater. Chem., 2011, 21, 3335-3345.

40 Y. Nie, L. Li and Z. D. Wei, Chem. Soc. Rev., 2015, 44, 21682201.

41 L. T. Qu, Y. Liu, J. B. Baek and L. M. Dai, ACS Nano, 2010, 4, 1321-1326.

42 H. R. Byon, J. Suntivich and Y. S. Horn, Chem. Mater., 2011, 23, 3421-3428.

43 X. D. Zhang, J. J. Zhang, J. Y. Zhao, B. C. Pan, M. G. Kong, J. Chen and Y. Xie, J. Am. Chem. Soc., 2012, 134, 11908.

44 Y. Zheng, Y. Jiao, J. Chen, J. Liu, J. Liang, A. J. Du, W. M. Zhang, Z. H. Zhu, S. C. Smith, M. Jaroniec, G. Q. (Max) Lu and S. Z. Qiao, J. Am. Chem. Soc., 2011, 133, 20116.

45 J. J. Duan, S. Chen, S. Dai and S. Z. Qiao, Adv. Funct. Mater., 2014, 24, 2072.

46 S. Wang, D. Yu and L. Dai, J. Am. Chem. Soc., 2011, 133, 5182. 47 S. Wang, D. Yu, L. Dai, D. W. Chang and J.-B. Baek, ACS Nano, 2011, 5, 6202.

48 L. Y. Zhang, C. X. Guo, Z. Cui, J. Guo, Z. Dong and C. M. Li, Chem.-Eur. J., 2012, 18, 15693.

49 J. N. Zheng, L. L. He, C. Chen, A. J. Wang, K. F. Ma and J. J. Feng, J. Power Sources, 2014, 268, 744-751. 